\title{
SDe

\section{Transdisciplinary System Science: Implications for Healthcare and Other Problems of Global Significance}

Azad M. Madni ${ }^{1}$, Daniel J. Epstein, Department of Industrial Engineering, Viterbi School of Engineering, University of Southern California, CA, USA, Email: azad.madni@usc.edu

doi: $10.22545 / 2010 / 0008$

( he past decade has witnessed transformational changes on a variety of scientific, technological, and societal fronts. On the scientific front, advances in system science methods, semantic integration techniques, complexity theory, and visual analytics are making it possible to address complex systems problems such as healthcare, climatology, and clean energy. On the technological front, there have been groundbreaking advances such as multicore processors, virtualization and cloud computing, and handheld platforms that have revolutionized the very nature of work. These advances are shaping the research and education agenda for the twentyfirst century. On the societal front, technology has transformed how people communicate, share information, socialize, and learn. Facebook and Twitter have transformed our modes and patterns of communication and expectations about the very nature of collaborative work. Crowdsourcing is becoming a popular means for rapid and cost-effective information acquisition, expertise location, and distributed problem solving. These advances have occurred none too soon in that the interconnectedness of todays world has made socio-technical problems increasingly more complex and unquestionably beyond the purview of every single discipline. Not surprisingly, this recognition has spurred the recent surge in interest in transdisciplinary system science research and education. At the heart of transdiscipinary system science are transdisciplinary thinking and collaboration, complex systems modeling and evaluation, and transdisciplinary system science education. This change in mindset is expected to not only enrich existing disciplines but potentially lead to the creation of entirely new disciplines. Specifically, transdisciplinary system science holds the potential of reaching beyond disciplinary boundaries to resolve incompatibilities and close knowledge gaps between disciplines. The challenge today is to energize communities and institutions of higher learning to address these challenges with a transdisciplinary mindset. This paper discusses transdisciplinary system science from the perspective of transforming the way we formulate problems, model complex systems, generate hypotheses, design interventions, conduct evaluations, disseminate findings, and continue to learn.

Keywords: transdisciplinarity, system science, complex systems, systems modeling, public health, obesity control. 


\section{Introduction}

While advances in social networking, collaboration, and crowdsourcing technologies have succeeded in "shrinkin" the world or, as Tom Friedman [1] puts it, making it "flat," realworld problems continue to grow in complexity. Not surprisingly, addressing these problems with techniques from a single discipline is becoming increasingly less viable. Today there is a growing recognition that it takes a combination of disciplines to create effective solutions to complex system problems. This fact has not gone unnoticed in the research and education communities as evidenced by the surge in interest in transdisciplinary research and education worldwide.

Despite its obvious allure, operationalizing transdisciplinarity for a particular problem domain (e.g., healthcare) has its share of challenges $[2,3,4,5]$. To begin with, academic and societal viewpoints differ. Fortunately, the academic research and business communities have recognized the need for transdisciplinary research and education frameworks [6]. For example, when it comes to public health, the $\mathrm{Na}$ tional Academies (National Academies, 2002) recommend moving from research dominated by a single discipline or a small number of disciplines to transdisciplinary initiatives. They define transdisciplinary research as involving broadly constituted teams of researchers that work across disciplines to develop and answer significant research questions. In these recommendations, transdisciplinary research implies the formation of research questions that transcend individual disciplines and specialized knowledge to solve public health research questions beyond the purview of any single discipline. In transdisciplinary public health research, different specialties seek to combine their expertise (and that of community members) to collectively define health problems and jointly pursue their solutions. The National Academies emphasize that the one qualitatively different and unique aspect of the transdisciplinary "process" is the holistic blending of expert and community inputs to produce greater integration across disciplines than exists today.

Transdisciplinary research implies a dialogue between the different disciplines and theories with a view to advancing both methodological and theoretical developments $[7,8,9,10]$. This characteristic sets transdisciplinary research apart from some forms of interdisciplinary research which tend to "assemble" different disciplines around particular themes and projects without making a commitment to changing the boundaries and relations between them.

Against the foregoing backdrop, the differences among intradisciplinary (or unidisciplinary), multidisciplinary, interdisciplinary, and transdisciplinary research can be identified. Rosenfield [11] defines these different types of research collaboration along a continuum. Unidisciplinary (or intradisciplinary, as I choose to call it) collaboration involves researchers from a single discipline working together to address a common problem. Multidisciplinary collaboration involves researchers from different disciplines working independently or sequentially, each from his or her own disciplinary-specific perspective, to address a common problem. Interdisciplinary collaboration involves researchers from different disciplines working jointly to address a common problem and although some integration of their diverse perspectives occurs, participants remain anchored in their own fields. Transdisciplinary collaboration involves researchers from different disciplines working jointly to create a shared conceptual framework that integrates and goes beyond discipline-specific theories, concepts, and approaches, to address a common problem. Table 1 compares and contrasts these various forms of research initiatives.

It is worth recognizing that transdisciplinarity has its roots in the increasing demand for relevance and applicability of academic research to societal challenges [12]. Not surprisingly, the two popular definitions of transdisciplinary research today center around academic research and societal challenges. The academic research-oriented definition characterizes transdisciplinarity as "a special form of interdisciplinarity in which boundaries between and beyond disciplines are transcended and disciplines as well as non-scientific sources are integrated." The societal challenge-oriented definition characterizes transdisciplinarity as "a new form of learning and problem-solving involving cooperation among different parts of society (including academia) to meet complex societal challenges. Solutions devised are a result of collaboration and mutual learning among multiple stakeholders." As can be seen from the preceding two definitions, there is no standard definition of transdisciplinarity. What is common to both, however, is the desire to achieve unity of knowledge. 
Table 1: Collaborative Research Typology (adapted from [6]).

\begin{tabular}{|c|c|c|c|c|}
\hline $\begin{array}{l}\text { Comparison } \\
\text { Factors }\end{array}$ & Intradisciplinary & Multidisciplinary & Interdisciplinary & Transdisciplinary \\
\hline Collaboration Scope & $\begin{array}{l}\text { - Among individuals } \\
\text { within a discipline }\end{array}$ & $\begin{array}{l}\text { - Among individuals } \\
\text { from different } \\
\text { disciplines }\end{array}$ & $\begin{array}{l}\text { - Among disciplines } \\
\text { through collaborators }\end{array}$ & $\begin{array}{l}\text { - Across and beyond } \\
\text { disciplines without } \\
\text { regard to disciplinary } \\
\text { boundaries }\end{array}$ \\
\hline Specific Focus & $\begin{array}{l}\text { - Deeper understanding } \\
\text { within a research field } \\
\text { (e.g., quantum } \\
\text { physics within } \\
\text { physics) }\end{array}$ & $\begin{array}{l}\text { - Achieving compatibility } \\
\text { in complex problem } \\
\text { solving through } \\
\text { collaboration }\end{array}$ & $\begin{array}{l}\text { - Creation of integrative } \\
\text { solutions potentially } \\
\text { resulting in mutual } \\
\text { enrichment of } \\
\text { disciplines }\end{array}$ & $\begin{array}{l}\text { - Finding hidden } \\
\text { connections among } \\
\text { knowledge elements } \\
\text { from different } \\
\text { disciplines }\end{array}$ \\
\hline Key Characteristics & $\begin{array}{l}\text { - Generally, study } \\
\text { same "research } \\
\text { objects," (e.g., } \\
\text { multiple branches of } \\
\text { modern physics) } \\
\text { - Tend to have } \\
\text { methodologies in } \\
\text { common } \\
\text { - Tight communications } \\
\text { - Mostly speak a } \\
\text { common language } \\
\text { - Add to the body of } \\
\text { knowledge (BOK) of a } \\
\text { branch/ discipline }\end{array}$ & $\begin{array}{l}\text { - Harmonize multiple, } \\
\text { occasionally } \\
\text { incompatible aspects } \\
\text { - Integration limited to } \\
\text { linking research } \\
\text { results } \\
\text { - Susceptible to } \\
\text { misunderstanding } \\
\text { (specialized } \\
\text { languages) } \\
\text { - Collaborators } \\
\text { occasionally unsure } \\
\text { about final resolution }\end{array}$ & $\begin{array}{l}\text { - Development of } \\
\text { shared concepts, } \\
\text { methods, } \\
\text { epistemologies for } \\
\text { explicit information } \\
\text { exchange and } \\
\text { integration } \\
\text { - Specialization causes } \\
\text { knowledge } \\
\text { fragmentation, } \\
\text { occasionally } \\
\text { contradictory } \\
\text { knowledge }\end{array}$ & $\begin{array}{l}\text { - Challenge the norm } \\
\text { and generate options } \\
\text { that appear to violate } \\
\text { convention } \\
\text { - Look at problems } \\
\text { from a discipline- } \\
\text { neutral perspective } \\
\text { - Employ themes to } \\
\text { conduct research and } \\
\text { build curricula } \\
\text { - Redefine disciplinary } \\
\text { boundaries and } \\
\text { interfaces } \\
\text { - Can produce an } \\
\text { entirely new discipline }\end{array}$ \\
\hline
\end{tabular}

\section{Transdisciplinary Research and System Science}

At the outset, it is worth recognizing the subtle differences between system science $[13,14,15]$ and transdisciplinary science. One of the objectives of system science is the unification of knowledge residing in different "worlds." In subtle contrast, transdisciplinary science is concerned withdiscovering hidden connections between different disciplines with a view to establishing a common platform for discourse among people from diverse disciplines. Peter Checkland [16] suggests that "what we need is not interdisciplinary teams, but transdisciplinary concepts; concepts which serve to unify knowledge by being applicable in areas which cut across the trenches which mask traditional academic boundaries." Norbert Wiener [17] was among the first to write about the growingly interconnected complex of concepts and models, and about ways of interaction among elements and organizations of complex situations and systems. These perspectives led to the notion of "transdisciplinary synthesis," potentially a new language of interconnected concepts and models applied to reasonably accurate descriptions of complex wholes or "multi-domain ontologies." However, while being cognisant of the ills of hyperspecialization, it is also important to be mindful of the fact that a theory of everything does not devolve into a "theory of nothing."

The emergence of transdisciplinary research has been several years in the making as societal problems continue to grow beyond the confines of a single discipline [6]. As noted earlier, transdisciplinary research requires collaboration beyond that addressed by intradisciplinary, multidisciplinary, and interdisciplinary research. Transdisciplinary research is characterized by collaborative interdisciplinary teams engaged in transdisciplinary thinking (i.e., thinking beyond the traditional disciplinary boundaries) to understand and fill knowledge gaps and reconcile incompatibilities that exist among disciplines.

Looking back a few decades, problems tended to be relatively well-circumscribed and amenable to analysis and solution approaches using methods 
from a single engineering discipline. Years later, led by the aerospace industry, the discipline of systems engineering was born. Systems engineering required people from different disciplines to collaborate to solve problems that were deemed unsolvable using techniques from within a single discipline. With the advent of systems engineering, the emphasis shifted fromapplying the right technique to solve a problem to identifying and bringing together the right mix of people from different disciplines to solve complex problems. This was the beginning of multidisciplinary problem-solving which has its roots in multidisciplinary collaboration.

Collaboration among people from different disciplines led to the identification of knowledge gaps and the recognition that some problems required making extensions to the contributing disciplines. Occasionally, entirely new disciplines (e.g., electromagnetics, biomechanics, cognitive engineering, behavioral economics) with new sets of concepts emerge from such collaboration, and become objects of research in their own rights. For example, electromagnetics resulted from the union of electronic and magnetic fields and potentials. Researchers from these two disciplines found that the movement of a charged object created a magnetic field. When this hidden connection between these two disciplines was discovered, it created an entirely new field-electromagnetics.

Along with cross-fertilization and cross-pollination among disciplines came the recognition that there were incompatibilities among disciplines arising primarily from differences in underlying assumptions and theoretical foundations. These differences, in part, stood in the way of knowledge unification across disciplinary boundaries. It is this recognition that leads to the realization that we need to transcend (i.e., go beyond) disciplines to fill in knowledge voids and harmonize disciplines. This new awakening provides the impetus for transdisciplinary collaboration as a means to achieve knowledge unification across disciplines and domains.

Transdisciplinary research is conducted by interdisciplinary teams working on complex problems requiring expertise in multiple disciplines and knowledge of different domains. The product of such collaboration, if successful, is not merely solutions to complex problem but also unification of knowledge from different domains and disciplines. Ultimately, the goal is unity of knowledge which includes not only knowledge associated with different disciplines but also knowledge between and across disciplines. Figure 1 shows how interdisciplinary collaboration can produce transdisciplinary concepts.

Transdisciplinary research stands to greatly benefit from a systems perspective and, more specifically, a system science perspective. Systems science is an interdisciplinary field of science that is concerned with the study of complex systems in nature, society, and other sciences. In this paper, systems science is used as a neutral term which subsumes a variety of systems-related fields such as systems theory, systems thinking, system dynamics and system modeling. Its focus is on holistic thinking and generative, iterative processes. Its generative nature leads to the creation of novel hypotheses which is in sharp contrast to reductionist approaches that begin with pre-determined, specific hypotheses. A variety of modeling approaches are subsumed within the system modeling rubric including system dynamic models, concept graphs, agent-based modeling, and macro-micro simulation models. The infusion of system science into transdisciplinary research provides the means to model complex problems, and use the model to: generate transdisciplinary hypotheses; inform and guide the development of interventions; develop evaluation criteria to assess the impact of interventions; analyze the sensitivity of interventions to sociocultural and environmental factors; and capture lessons learned with contextual information.

Systems science also stands to benefit from transdisciplinary research in important ways. Transdisciplinary thinking and collaboration seek to challenge traditional disciplinary boundaries with the intent of uncovering hidden connections. Such discoveries can potentially expand the discourse about the complex system and lead to enhancement of system models. The enhanced models provide a platform for the generation of novel transdisciplinary hypotheses and construction of transdisciplinary interventions.

What distinguishes transdisciplinary system science-oriented thinking from traditional reductionist approaches is that transdisciplinary thinking emphasizes lateral or associative thinking [18], often relying on metaphors and analogies to enhance problem understanding. In particular, transdisciplinary approaches employ integrative (or synthetic) problem solving as opposed to analytic problem solving typically employed by reductionist approaches $[6,19]$. Table 2 compares and contrasts analytic and synthetic problem solving that underlie tradi- 


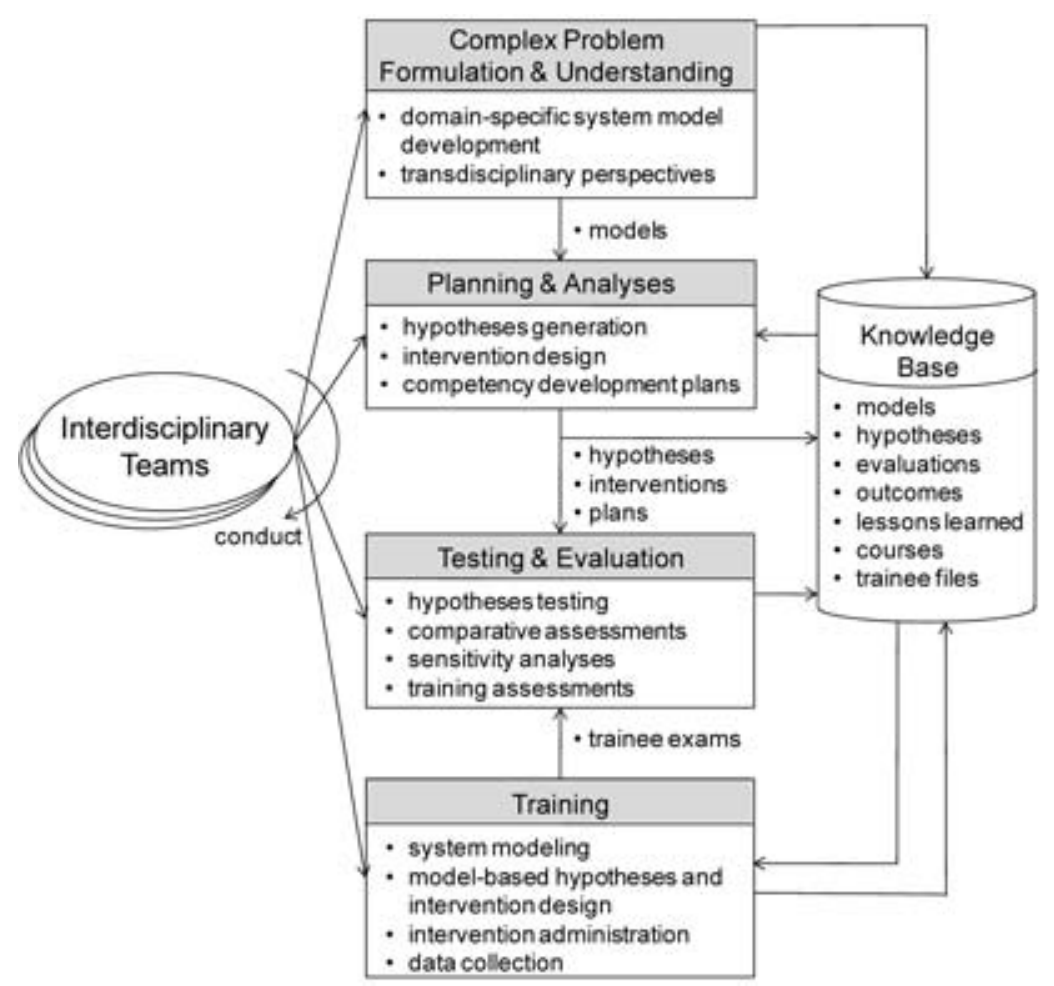

Figure 1: Transdisciplinary Concepts Emerge From Interdisciplinary Collaboration.

Table 2: Analytic versus Synthetic Problem Solving [19].

\begin{tabular}{|l|l|l|}
\hline \multicolumn{1}{|c|}{ Comparison Factors } & \multicolumn{1}{|c|}{ Analytic Problem Solving } & \multicolumn{1}{c|}{ Synthetic (Integrative) Problem Solving } \\
\hline Thinking Style & Reductionist & Holistic \\
\hline Emphasis & Solve by "divide and conquer" strategy & Understand and explain \\
\hline Solution Strategy & $\begin{array}{l}\text { Compose whole solutions from partial } \\
\text { solutions }\end{array}$ & $\begin{array}{l}\text { Whole solution greater than the some of the } \\
\text { partial solutions }\end{array}$ \\
\hline Shaping Mechanism & Command and control & Incentivize and influence \\
\hline Examples & Classification, diagnosis, troubleshooting & Planning, design, architecting \\
\hline Representative Systems & Aircraft, ship, tank, automobile, PDA & Internet, healthcare, energy grids \\
\hline
\end{tabular}

tional (reductionist) and transdisciplinary (holistic) approaches.

\section{Stimulating Transdisciplinary Thinking and Modeling}

Transdisciplinary research requires a transdisciplinary mindset [6]. A transdisciplinary mindset is one that is open to questioning disciplinary assumptions, and one that is willing to reach out to other disciplines to find solutions to problems [6]. Table 3 presents some of the key characteristics of a transdisciplinary mindset.
In recent years, researchers are turning totransdisciplinary research frameworks and system-based methodologies to overcome the limitations of todays research infrastructure. In particular, the National Institute of Child Health and Human Development, National Cancer Institute, Office of Behavioral and Social Sciences Research, and National Institute of Health are actively soliciting research grant applications. For example, NICHHD is interested in establishing a center of excellence for childhood obesity research and training based on a transdisciplinary system science-oriented framework and methodologies with the intent of capturing 
Table 3: Characteristics of Transdisciplinary Mindset (adapted from [6]).

- Actively look for and exploit synergies among disciplines

- e.g., decision theory and artificial intelligence

- Seek out appropriate analogies that help with problem understanding and problem solving

- e.g., biological analogy exploitation - human immune system as a model for cybersecurity

- Frame the problem in a larger context to open up collaboration scope

- e.g., BMW's boxfish-like concept car was a result of collaboration between engineers and marine biologists

- Examine the problem as an outsider to develop new perspectives

- looking beyond entrenched thinking can open up the option space (i.e., possibilities)

- Formulate the problem from different perspectives to gain novel insights

- perspectives could include technical, organizational, social, cultural, and environmental

- Envision outcomes to determine what incentives to apply and what constraints to relax

- a "reality check" can cause the relaxation of constraints imposed by an entrenched mindset

- Strive for semantic interoperability among disciplines

- develop multi-domain ontologies to "smooth out" seams among disciplines

- reconcile assumptions and theories across disciplines (to the degree possible)

- create a shared vocabulary to address complex problems

- relax disciplinary boundaries to accommodate new concepts

- Explicitly formulate transdisciplinary tradeoffs by reaching beyond disciplinary boundaries

- encourage team to view problems in a new light ("open mental locks")

- Employ model-based approaches to generate transdisciplinary hypotheses and interventions

- develop complex systems models using, for example, system dynamics modeling

- exercise these models to generate hypotheses and interventions and define metrics

etiological complexity of childhood obesity and the potential impact of environmental and/or policy interventions. Transdisciplinary system science is being viewed as the means to go beyond traditional disciplinary boundaries and thereby overcome the limitations of existing research infrastructures. In particular, systems-oriented research is viewed as key to overcoming reductionist thinking and generating sustainable solutions within the broader social, cultural, and economic environment.

In healthcare, transdisciplinary collaboration is critical to pushing the boundaries of intervention approaches and, in so doing, contribute to and expand the frontiers of existing science and/or create new science $[20,21]$. In particular, transdisciplinary collaboration is key to the formulation of crossdisciplinary, cross-level research hypotheses that, in turn, enable the creation of effective structural, environmental, or policy-related interventions. Ultimately, cross-disciplinary, cross-level hypotheses are key to creating superior structural, environmental, and policy level interventions that are key to realizing sustainable solutions in the public health arena.
In this regard, systems modeling using systems dynamics, plays a key role in breaking the traditional "linear systems thinking" mindset that invariably attenuates weak effects of meso-level interventions on system behavioral trends. From an epistemological perspective, traditional approaches tend to bias research based on linear cause-effect models on the most proximate cause-effect relationships and treat the distal effects as sources of noise. However, from a systems perspective in which one can have nonlinear feedback relationships, weak relationships within a feedback loop can accumulate over time to eventually become the main driver of system behavior [22]. This is "emergent" behavior which cannot be reduced to and reflected in the properties of the individual subsystems/components, but can only be understood by viewing the system as a whole (i.e., holistically). This phenomenon complicates the evaluation of policy interventions. Consequently, new methods, processes, and tools are needed to handle the complexities of the system. For example, in the world of childhood obesity control, without such methods it is rarely possible to have an adequate 
theory for generating hypotheses about the complex ways that community level interventions and their sequence and timing can affect outcomes over time.

When a complex system is viewed from a system dynamics perspective, the system is characterized as a set of coupled, nonlinear differential equations. With this characterization, it has been found that most parameters exert some influence, however weak, on the magnitude of the trends, and only a few parameters exert sufficient influence to actually alter the qualitative shape of the trajectory being studied. It is interesting to note that most model parameters can vary significantly ( $50 \%$ in either direction) without qualitatively impacting the shape of the system trajectory [23]. It is equally important to note that the influence of feedback mechanisms changes over time; that is, the system evolves thereby confounding previous explanations of behavior using traditional methods. From an epistemological perspective, this does not mean that all other mechanisms are not contributing to shaping behavior in some way, but rather that their contributions are small. For example, with respect to childhood obesity control, these minor mechanisms are likely to influence how fast obesity trends are increasing, but are not sufficiently influential to determine whether obesity trends show an increase or decrease in childhood obesity [23].

\section{Transdisciplinary System Science (TSS) Application to Public Health}

\subsection{Transdisciplinary System Science Framework}

Healthcare has been described as a complex adaptive system $[24,25,26,27,28]$ that is not amenable to being managed by traditional command and control methods. Instead, this complex adaptive system can be shaped and influenced through appropriately designed interventions and incentives. These interventions and incentives show up in the form of policy changes, new regulations, promotional programs, and in the form of guidance counseling, therapy, advertisements, and training at the community and individual levels. The TSS Framework presented in this paper is an example of viewing a complex enterprise (e.g., healthcare) through a transdisciplinary lens, and analyzing and evaluating the behavior of the enterprise to a variety of interventions and incen- tives through a system science lens. Figure 2 presents the overall TSS Framework. The key elements of this framework are discussed next.

TSS Blackboard. This is the shared global database that mediates information among the various stakeholders, and maintains status of plans, hypotheses, interventions, evaluations, and outcomes that comprise the TSS Framework (Figure 2). The blackboard architecture, based on the "cooperating specialists" paradigm [19] ensures that the various users of the TSS Framework interact solely with the blackboard without having to worry about the other "specialists" using or contributing to the blackboard. This feature makes the TSS Framework totally scalable while ensuring that the addition/departure of a "specialist" does not require changes to the other elements or disrupt the integrity of the overall architecture. This feature is especially desirable as new partners come into or leave the overall enterprise.

Participating Entities. The participating entities range from individuals, advisory groups, and officials, to national and international organizations and programs. These entities contribute resources and information to the TSS Framework and draw on informational resources provided by the framework.

Public Policies and Communication Strategies. These include public policy changes, new communication strategies, and behavior change incentives. These strategies are intended to accomplish short-term behavior changes, produce longer-term behavioral shifts, and, ultimately, contribute to sustainable health and lifestyle changes.

Tactical Behavior Change Interventions. These interventions, focused on short-term changes, employ strategies that take the form of some kind of stimulus that "jumpstarts" the process of change, where the desired change could be, for example, in eating habits and physical activities.

Strategic Behavior Change Interventions. These interventions, focused on achieving longerterm changes, employ incentives that ensure that the community and population can "stay the course." Examples of staying the course are staying on a healthy diet and exercise regimen that can ultimately become ingrained in the community or population.

Training Programs. These initiatives, that are tailored for each community and population, are 


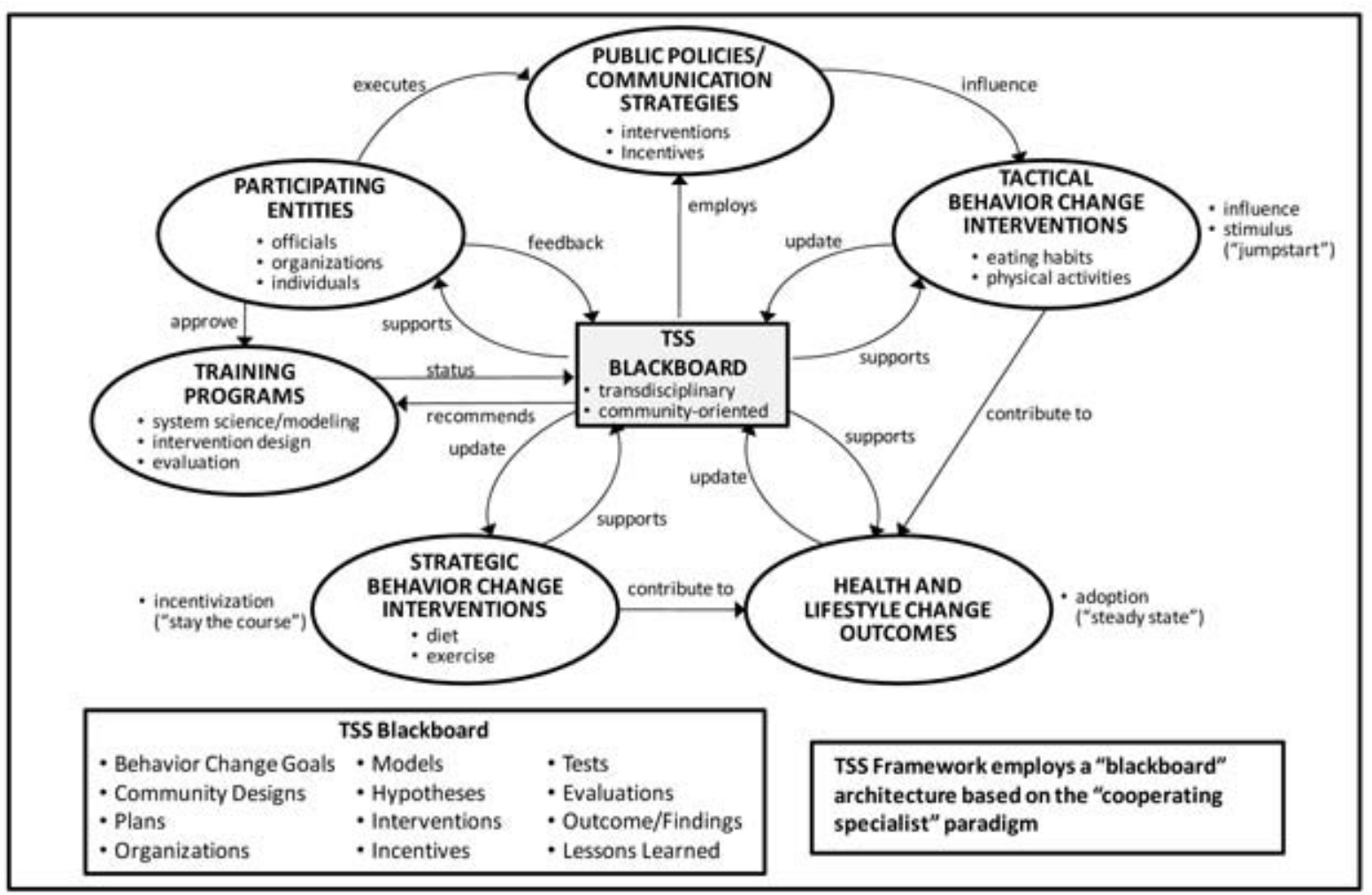

Figure 2: The TSS Framework: Coordination Perspective.

intended to exploit a variety of methods and media to teach community members, trainers, community organizers, health advocates, family members in healthful lifestyle practices. They are also intended to teach public health domain system modelers in a variety of modeling methods (e.g., concept graphs, system dynamics, agent-based simulations, Markov models, macro-micro simulations) that can inform the development of transdisciplinary research hypotheses, and transdisciplinary interventions. The training programs are also intended to target administrators and community organizers responsible for administering interventions and evaluating their impact.

\section{Health and Lifestyle Change Outcomes.} These are sustainable results that reflect the adoption of the behavioral changes by communities and populations. These imply that the communities/populations have reached "steady state" and are highly unlikely to revert to old bad habits and behaviors that contribute to, for example, childhood obesity.

\subsection{TSSF Application to Complex Healthcare Problem}

Figure 3 presents the development and application perspectives of TSSF for a complex healthcare problem (e.g., childhood obesity control). As shown in Figure 3, starting with a basic set of enabling modeling techniques and tools from different disciplines (e.g., system dynamics, computer science, cognitive science, social sciences), we can combine and apply these tools in new and novel ways to create the systems science "toolbox." This toolbox is used to create a systems model for the complex problem (e.g., childhood obesity control). This model provides the basis for testing and evaluating various transdisciplinary hypotheses and interventions. The results of model-based test and evaluation are used to tailor the training toolbox that is used to teach systems modeling, transdisciplinary hypotheses generation, intervention design and implementation, intervention administration, and outcome evaluation. The training toolbox supports both web-based and instructor-led training strategies. The trainees include local community organizers and community members involved in mitigating complex health prob- 


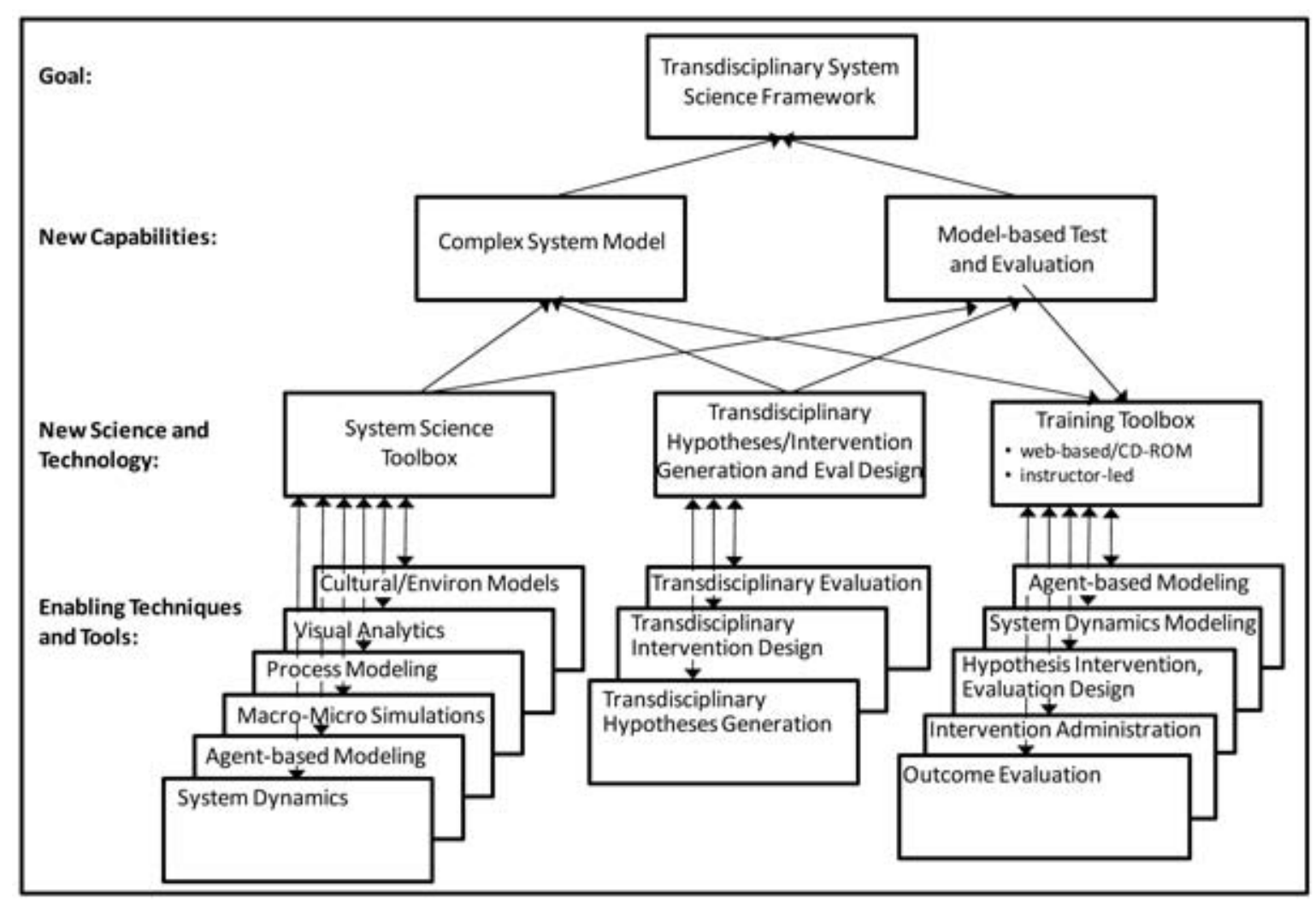

Figure 3: TSS Framework: Development and Application Perspective.

lems.

\subsection{The TSS Process Model}

The TSS process begins with Problem Formulation followed by Systems Modeling and Analysis (Figure 4). The outputs of these activities are system models, which inform and guide transdisciplinary hypotheses generation, intervention design and implementation, data collection, and impact evaluation. The latter prompts formulation of new problems, generation of new hypotheses, and design of new interventions at multiple levels. As this closed-loop TSS process is undertaken, transdisciplinary system science assets are generated and persistently stored in the TSS Assets Library (TSSAL). Concurrently, training material is developed for teaching communities (local and international) in how to formulate problems, how to model systems using a variety of techniques (e.g., concept graphs, system dynamics models, agent-based models, and parametric models), how to design interventions at multiple levels (policy, socio-cultural, biological), and how to collect data and evaluate results. The evaluation results can initiate a new cycle starting with new problem definition.

The TSSAL contains models, tools, hypotheses, interventions, stakeholders, lessons learned about specific interventions in terms of their immediate, intermediate, and long-term impacts. The lessons learned are intended to inform and guide users of the TSSF in undertaking other major public health initiatives. The TSSAL also contains training content and courseware. The courseware includes learning objectives, instructional strategies, tests/quizzes and evaluation metrics. The content is stored as shareable content objects (SCOs) in accord with the SCORM standard. These objectives can be reused and repurposed for a variety of training applications [29], thereby saving time and money in training development. In short, the vision of TSSAL is that of a repository of TSS models, tools, facts, experiments, and outcomes, that can be institutionalized internationally to support global health initiatives. 


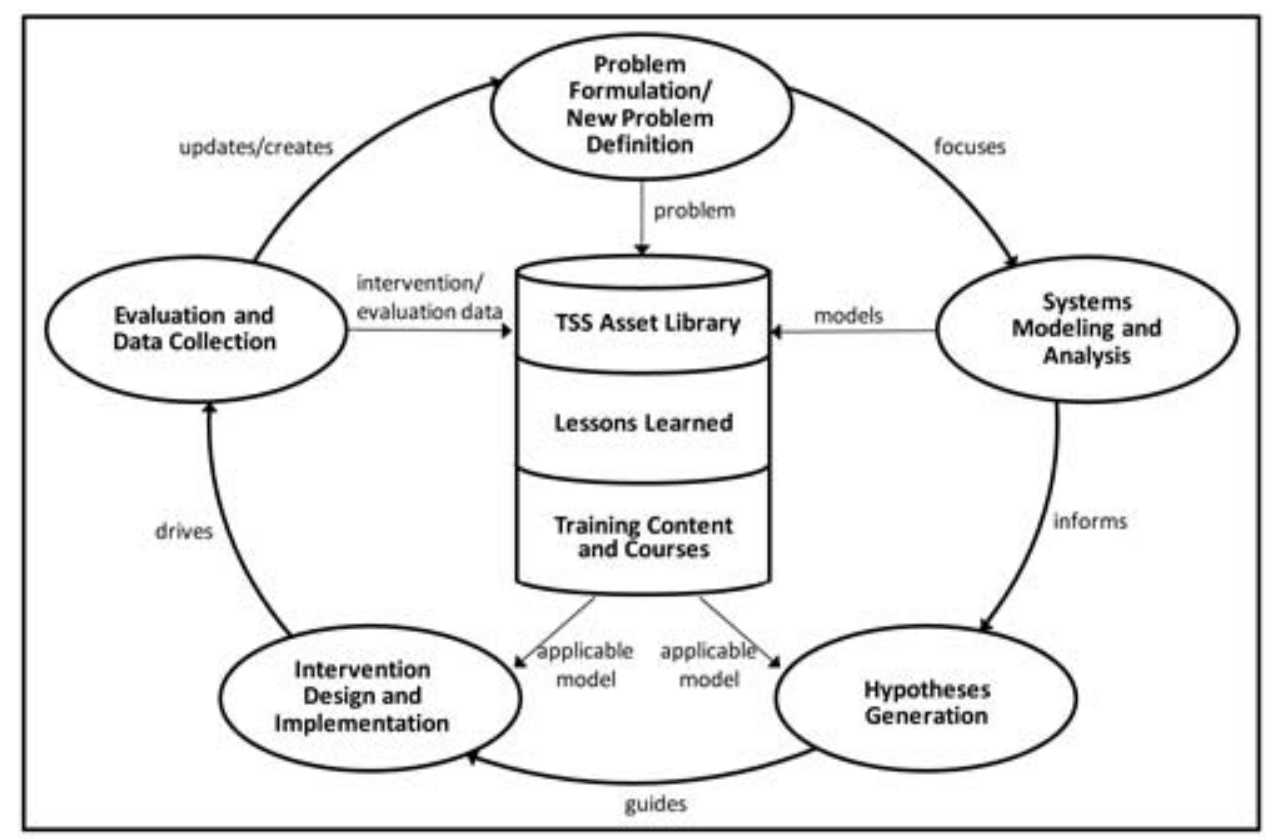

Figure 4: TSS Model-driven Research and Training Development Process.

\subsection{Systems-oriented Model of Childhood Obesity}

Figure 5 presents a systems-oriented, multi-level, multi-perspective model for childhood obesity problem analyses and intervention design to achieve desired health outcomes. The model is based on the recommendation of Glass and McAtee [30], who note that since social factors (e.g., social inequality, poverty) are difficult to study from a traditional epidemiologic perspective, they ought to be viewed as risk regulators or influencers of obesity-related behaviors (at the individual, community, and public policy levels). For example, food distribution systems alter obesity likelihood at the population level that, in turn, lead to different rates of obesity. The challenge is to identify the key risk regulators within the social, physical, cultural, and economic environments that influence obesity. The concept of risk regulators overcomes the lack of clarity about the key obesity drivers, their locations, and the determination of the optimal intervention points. The multiple levels of the model are intended to acknowledge both the macro-level forces and the local environmental factors that govern/influence eating habits and physical activity. Huang et al [31] explain this influence chain by describing the temporally and spatially distal forces at the macro level that cascade through organizations, through systems of food distribution, through policies and pricing, and eventually shape the perceptions of people. Examples of the intervening variables for obesity include cultural norms, social networks, local food availability, food prices and taxes, physical activity amenities, psychosocial stress, and economic conditions. These factors can potentially act through neurologic/epigenetic regulatory pathways to affect behavior and generate feedback loops to higher levels in the system [31].

\subsection{Setting Up a Transdisciplinary Research and Training Organization}

Transdisciplinary collaboration is the hallmark of a transdisciplinary research organization. Setting up such an organization requires incorporating transdisciplinary perspectives into all aspects of the organization including organization design, research problem formulation, hypotheses generation, intervention design, intervention efficacy evaluation, and training. Transdisciplinary collaboration involves multiple stakeholders and multiple specialists with different expertise areas (e.g., business operations, system science, system modeling, obesity control, policy design, environmental sciences, social sciences, experimental design) working closely to: a) develop the vision and mission statement for the research and training organization; b) architect the organization to facilitate transdisciplinary research and 


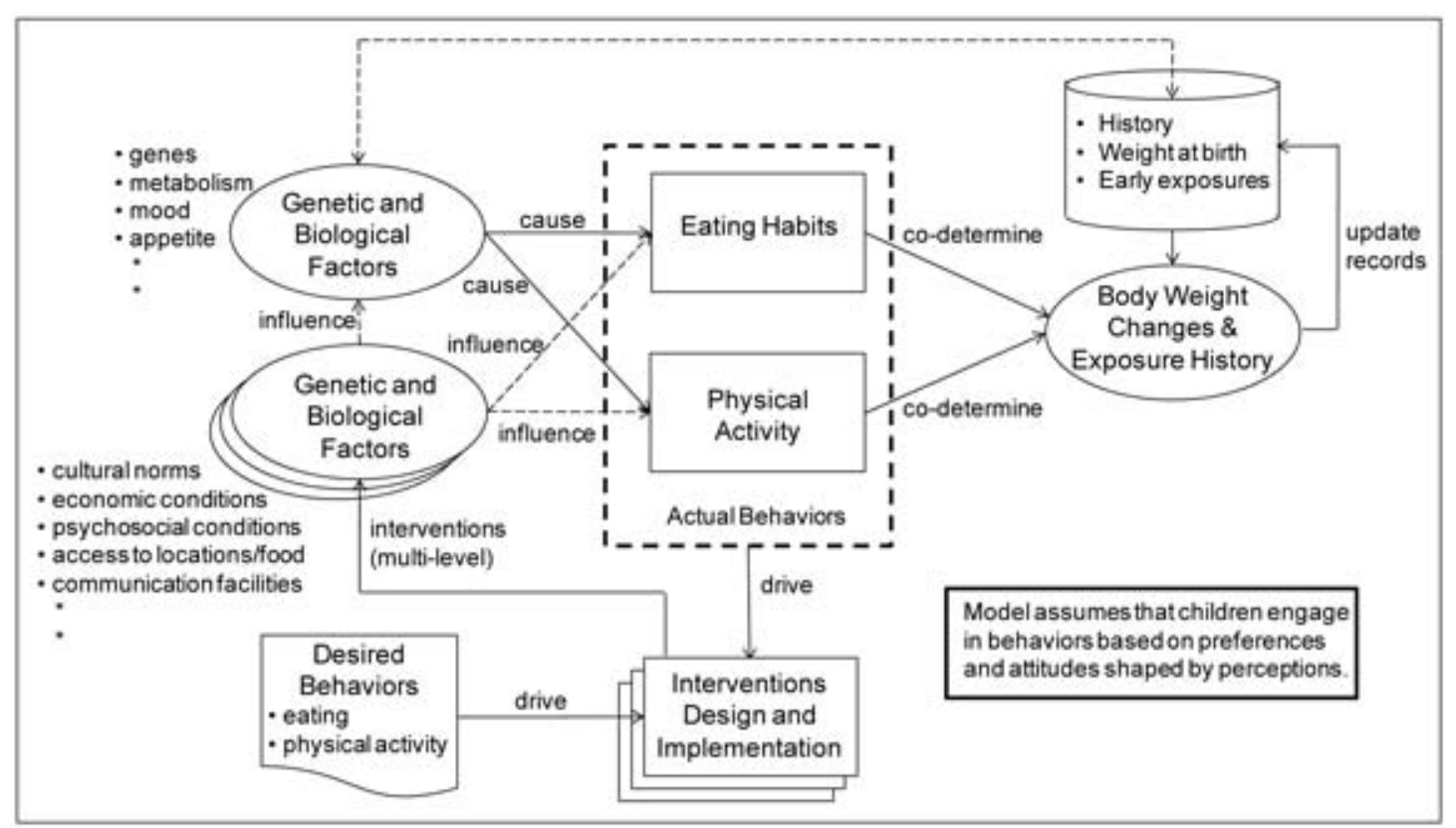

Figure 5: Systems-oriented Multi-level Model for Childhood Obesity Control.

education; c) conduct domain analysis; d) model the complex system at the community level; e) generate transdisciplinary hypotheses; f) design and implement transdisciplinary interventions; and $g$ ) evaluate efficacy of the interventions, and achievement of the organizations objectives. The infusion of transdisciplinary systems thinking and collaboration in the design and operation of a research and education organization is presented next.

Vision and Mission Definition involves developing the transdisciplinary research and education agenda of the organization and the impact it is expected to have in both the short-term and longerterm on: a) the organizations goals and objectives; and b) health outcomes (e.g., impact on childhood obesity rates).

Transdisciplinary Organization Design involves creating an organization architecture that lends itself to supporting transdisciplinary research and education. This includes the leadership of the organization, the creation of distributed collaborative teams, the emphasis on capacity building including training, setting stretch goals that push the boundaries of intervention approaches, and laying the groundwork for a sustainable area of new science.

Domain Analysis involves analyzing the target problem domain form transdisciplinary perspectives.
Thus, the problem domain of childhood obesity prevention and control would be analyzed from a process perspective, socio-cultural perspective, environmental perspective, economic and educational perspective, epidemiology perspective, and intervention design perspective. As importantly, the analysis of interactions around macroenvironmental, macrosocial, and biological factors would be studied from a transdisciplinary perspective.

System Modeling involves modeling the complex system at different levels of abstraction and from different perspectives using a variety of systems science approaches and system modeling tools (e.g., concept graphs, system dynamics, process modeling, behavioral modeling at individual, organizational, and community levels) and in the presence of a variety of modifiers (e.g., social, cultural, environmental, economic, educational). The overall purpose of system modeling is to: a) enable the simultaneous examination of influences of a wide range of biological and socio-environmental factors on obesity behavior and outcomes; b) enable the evaluation of downstream impacts of environmental/policy interventions; and c) provide content for training a cadre of system scientists to address complex socio-technical problems in various significant domains such as public health and energy.

Iterative Hypotheses Generation involves em- 
ploying the complex system model to generate transdisciplinary hypotheses spanning a variety of interventions (e.g., environmental and policy) including training. The iterative nature of transdisciplinary hypotheses generation is intended to extend disciplinary boundaries and occasionally uncover hidden connections among knowledge elements from different disciplines, thereby providing new and useful insights for problem understanding and intervention design.

Iterative Intervention Design and Implementation involves exploiting the system model to develop effective interventions (e.g., environmental, policy, training) for a particular environment (i.e., geography, demographics, economic status, social and environmental conditions), and implementing the interventions in a culturally-aware, environmentallyconscious fashion.

Evaluation encompasses intervention evaluation (i.e., efficacy of the intervention in the target environment), training evaluation (i.e., improvements in trainee populations ability to model, design interventions, conduct surveys, and evaluate impacts), and organizational evaluation (i.e., the evaluation of an organization's ability to make its target objectives). The latter include trainee throughput, intervention outcomes (short-term, long-term), as well as an organization's ability to generate sustainable revenue streams through research, operation and training.

Curriculum Design and Training encompasses creating the right content (i.e., training material) and training strategies to teach target populations about how to engage in tarnsdisciplinary thinking, design communities, generate plans, model complex systems at various levels (e.g., the community level), generate transdisciplinary interventions, develop evaluation questionnaires and schemes, and develop evaluation metrics.

\section{Towards a Transdisciplinary System Science Research and Education Agenda}

The previous section presented the key concepts behind a transdisciplinary system science research framework and an illustrative example. This section provides strategies for creating a transdisci- plinary system science-oriented research and education agenda.

\subsection{TSS Research Agenda}

A research agenda for TSS research needs to be driven by problems of high complexity and scale that elude traditional approaches [6,32]. Preferably, these problems should be of global significance to garner international attention. Such problems tend to be complex socio-technical problems that span multiple disciplines, domains, societies, and cultures. They invariably require harmonizing terminologies across the contributing disciplines. Some of the earlier work in transdisciplinary education and research in the engineering disciplines was in relation to design and process science [33, 34]. Examples of such problems are disaster response, childhood obesity prevention and control, and global security and safety. The fourteen Grand Challenges of the National Academy of Engineering (http://www.engineeringchallenges. org/ cms/challenges.aspx) all involve complex sociotechnical problems at some level. Once such problems have been formulated from different perspectives, the relevant disciplines that potentially contribute to their solution need to be identified and researchers from the relevant disciplines assembled and incentivized to participate and stay the course. Thereafter, interdisciplinary collaboration among these researchers needs to begin. This process can potentially move disciplinary boundaries, resolve incompatibilities among disciplines, and occasionally result in enhancing theories. The resultant body of knowledge (BOK), after verification and validation by transdisciplinary teams, can be incorporated into the TSS educational agenda.

\subsection{TSS Education Agenda}

The TSS education agenda, in large part, "flows" from the TSS research agenda. It begins with the delineation of complex system characteristics that are beyond traditional intradisciplinary and multidisciplinary approaches. It needs to harness findings from interdisciplinary research to define new concepts and topics for inclusion in the curricula of the contributing disciplines. Thereafter, potential barriers to transdisciplinary education need to be identified and discussed along with the role of specific technologies (e.g., semantic technologies) to potentially overcome these barriers. Finally, a set 
of canonical problems that requires the creation, use, refinement and deployment of transdisciplinary "bridges" (e.g., multi-domain ontologies) needs to be included in the overall educational agenda to increase the students understanding of problems requiring TSS solutions.

\section{Conclusions}

As science moves deeper into the workings of the universe, we will increasingly develop models and methods that unite disciplines. Electromagnetics, biostatistics, cognitive engineering, psychophysiology and medical informatics are but a few examples of this phenomenon. Today, we can engineer materials atom by atom, working very nearly at the boundary between matter and energy. At this level, disciplinary distinctions become almost arbitrary as physics, chemistry, biology and engineering begin to converge upon shared possibilities [35]. The "promise of converging spaces" can be profound and far-reaching. Some of the pressing challenges that can be addressed through such convergence include: mitigating the damage we inflict on the environment; producing new materials to support the rapid development of worldwide infrastructure, defending ourselves against escalating chemical-biological threats; and increasing computing power while reducing size and cost. Such pressing priorities are beyond the purview of a single discipline, a single institution, or even a single society or culture.

This is the essence of the TSS approach. However, the promise of transdisciplinarity comes with its fair share of challenges. To begin with, any transdisciplinary approach requires "going beyond the laboratory" and into the realm of politics. No far-reaching reform or advance is possible without getting into the realm of politics. Very simply, politics is the process by which humans express desires, establish priorities, and allocate resources [35]. The key question, of course, is whether politics will advance or hinder the advance of promising technologies. Clearly, while these types of questions are addressed in the realm of politics, scientists must step forward to represent the possibilities that may otherwise go unvoiced, unnoticed, or worse yet, misunderstood.

For TSS research discussions to go beyond the abstract into making a difference to pressing issues in the realworld, researchers need to initially identify regional problems and issues at various scales and, after demonstrable successes, elevate their sights to issues of national and global significance. Regional issues can be identified in a variety of venues such as energy conservation and use, environment management systems, global climate change management, healthcare, sustainable development, and educational reform. Once such problems have been identified, an appropriate mix of disciplinary breadth and depth can be specified based on the theme, issue or problem addressed. It is almost inevitable that addressing such socio-economic and socio-political problems will require linking specific scientific disciplines with humanities.

However, realizing a TSS educational curriculum requires several changes at the content, instruction, and institutional levels. To begin with, course content needs to be focused on those real problems and issues that are not amenable to solution or resolution from within a single discipline and that require interdisciplinary teams. Second, there is a need for faculty members with an open mind who are willing to look for and discern emergent connections among disciplines and develop new insights. Third, educational institutions need to not only be accepting of this paradigm shift but, in fact, create an environment that attracts and incentivizes TSS educators and researchers. Fourth, the curricula need to be viewed not merely from the perspectives of depth and breadth but from a thematic perspective. The syllabus needs to be theme-focused, integrated with the appropriate disciplines, and at a level of depth and breadth consistent with the theme. Fifth, since the internet has dramatically facilitated the conduct of transdisciplinary research [36], it should be exploited in web-based learning and distance learning programs. Finally, concrete examples of themerelated transdisciplinary solutions and experiences need to be covered to develop transdisciplinary thinking skills. In conclusion, the time has come for us to begin exploiting the "flatness" of this world with open minds and a commitment to TSS research and education, the next frontier in the intellectual and societal growth of human kind.

\section{References}

[1] Friedman, T.L., 2005. The World is Flat: A Brief History of the Twenty-first Century. Copyright Thomas L. Friedman.

[2] Barabasi, A.L., 2007. Network medicinefrom obe- 
sity to the "diseasome." New England Journal of Medicine, 357, pp. 404407.

[3] Ackoff, R.L., 1961. Systems, organizations and interdisciplinary research. In: Eckman DP, ed. Systems research and Design, New York: Wiley.

[4] Nicolescu, B., La Transdisciplinariet, Paris, France: Rocker, International Congress. What university for tomorrow? Towards a transdisciplinary evolution of the university, Locarno, Switzerland, April 30 May 2.

[5] Provan, K.G., Clark, P., Huerta, T., 2008. Transdisciplinarity among tobacco harm reduction researchers: a network analytic approach. American Journal of Preventive Medicine, 35(2S), pp. 173181.

[6] Madni, A.M., 2007. Transdisciplinarity: Reaching beyond Disciplines to Find Connections. Journal of Integrated Design and Process Science, 11(1), pp. $1-11$.

[7] Fairclough, N., 2003. Analyzing Discourse: Textual Analysis for Social Research. Routtedge.

[8] Fairclough, N., 2005. Critical discourse analysis in transdisciplinary research on social change: transition, re-scaling, poverty and social inclusion.

[9] Best, A., Moor, G., Holmes, B., et al., 2003. Health promotion dissemination and systems thinking: towards an integrative model. American Journal of Health Behavior, 27(3S), pp. 206216.

[10] Gibbons, M., Limoges, C., Nowotny, H., Schwartzman, S., Scott, P., Trow, M.,1994. The New Production of Knowledge. The Dynamics of Science and Research in Contemporary Societies (Sage Publications.

[11] Rosenfield, P.L. 1092. The potential of transdisciplinary research for sustaining and extending linkages between the health and social sciences. Social Sciences in Medicine, 35, pp. 13431357.

[12] Nicolescu, B.,202. Manifesto of Transdisciplinarity. SUNY Press, New York.

[13] Green, L.W., 2006. Public health asks of systems science: to advance our evidence-based practice, can you help us get more practice-based evidence? American Journal of Public Health, 96, pp. 406409.

[14] Leischow, S.J., Best, A., Trochim, W.M., Clark, P.I., Gallagher, R.S., Marcus, S.E., and Matthews, E., 2008. Systems Thinking to Improve the Publics Health. American Journal of Preventive Medicine, 35(2S), pp. 196-203.
[15] Leischow, S.J, Milstein, B., 2006. Systems thinking and modeling for public health practice. American Journal of Public Health, 96, pp. 403405.

[16] Checkland, P., 1981. Systems Thinking, Systems Practice.John Wiley \& Sons, Inc., New York. (Practice and Philosophy of Systemic Management).

[17] Weiner, N., 1948. Cybernetics, or Control and Communication in the Animal and the Machine. New York: John Wiley \& Sons, Inc.

[18] Madni, A.M., Brenner, M.A., Costea, I., MacGregor, D., Meshkinpour, F., 1985. Option Generation: Problems, Principles, and Computer-Based Aiding. Proceedings of 1985 IEEE International Conference on Systems, Man, and Cybernetics, Tucson, Arizona, November, pp 757-760.

[19] Madni, A.M., 1988. The Role of Human Factors in Expert Systems Design and Acceptance. Human Factors Journal, 30(4), pp. 395-414.

[20] Midgley, G., 2006. Systemic intervention for public health. American Journal of Public Health, 96, pp. 466472 .

[21] National Cancer Institute. Greater than the sum: systems thinking in tobacco control. Tobacco Control Monograph No. 18. Bethesda MD: USDHHS, NIH, National Cancer Institute. NIH Pub. No. 06-6085, 2007. http://dccps.nci.nih.gov/TCRB/monographs/18/ index.html, accessed: June 11, 2010.

[22] Senge, P.M., 1994. The fifth discipline: the art and practice of the learning organization. New York: Doubleday.

[23] Hovmand, P.S. and OSullivan, J., 2008. Lessons from an Interdisciplinary System Dynamics Course, System Dynamics Review, 24(4), pp. 479-488.

[24] Stokols, D., Hall, K.L., Taylor, B.K., Moser, R.P., 2008. The science of team science: overview of the field and introduction to the supplement. American Journal of Preventive Medicine, 35(2S), pp. S77S89.

[25] The Future of Public Health in the 21st Century. Committee on Assuring the Health of the Public in the 21st Century, 2002, The National Academies.

[26] von Bertalanffy, L., 1950. The theory of open systems in physics and biology. Science, 11, pp. 2329.

[27] Stokols, D., Fuqua, J., Gress,J., Harvey, R., Phillips, K., Baezconde-Garbanate, J.U., Palmer, P., Clark, M.A., Colby, S.M., Morgan, G., Trochim, W., 2003. Evaluating Transdisciplinary Science, Nicotine \& Tobacco Research, 5, pp. 21-39. 
[28] Sterman, J.D., 2006. Learning from evidence in a complex world. American Journal of Public Health, 96 , pp. 505514.

[29] Madni, A.M., 2011. Towards a Generalizable AidingTraining Continuum for Human Performance Enhancement. to be published in INCOSE Journal of Systems Engineering, Volume 14, Number 1, 2011.

[30] Glass, T.A., and McAtee, M.J., 2006. Behavioral science at the crossroads in public health: extending horizons, envisioning the future. Social Science Medicine, 62(7), pp. 1650-1671.

[31] Huang, T., Drewnowski, A., Kumanyeka, S.K., Glass, T.A., 2009. A System-oriented Multi-level Framework for Addressing Obesity in the 21st Century. Preventing Chronic Disease, Public Health Research, Practice, and Policy, 6(3).

[32] Tharp, T. and Zalewski, J., 2001. Economics and Software Engineering: Transdisciplinary Issues in Research and Education. Proceedings of the Workshop on Global Transdisciplinary Education, Research and Training, pp. 6-11.

[33] A. Ertas,Tanik, M. M., and Maxwell, T. T., 2000. Transdisciplinary Engineering Education and Research Model. SDPS Transactions: Journal of Integrated Design and Process Science, 4(4), pp. 1-11.

[34] Tanik, M.M. and Ertas, A., 1997. Interdisciplinary Design and Process Science: A Discourse on Scientific Method for the Information Age. Journal of Integrated Design and Process Science, 1(1), pp. 78-94.

[35] Upham, S.,2006. Convergent Technologies and Fundamentalist Ideologies: The Possibilities and Politics of the 21st Century. Keynote Address, Ninth World Conference of Integrated Design and Process Technologies, June 2006.

[36] Hunsinger, J., 2005. Toward a Transdisciplinary Internet Research. The Information Society, 21(4), pp. 277-279.

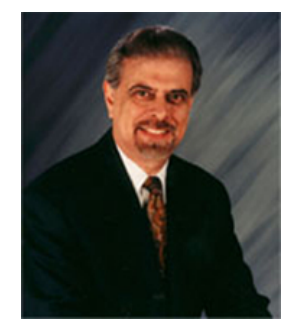

Azad M. Madni is the Professor and Director of the Systems Architecting and Engineering Program, Daniel J.
Epstein Department of Industrial \& Systems Engineering at the University of Southern California. Dr. Madni is internationally recognized for his pioneering research and technology innovations in modeling and simulation, process-aware systems, human-system integration, intelligent tutoring, concurrent engineering, and distributed training. He is the recipient of several prestigious, national awards including the SBAs 1999 National Tibbetts Award for California for excellence in technology innovation, Mass Mutual and US Chamber of Commerces 2000 Blue Chip Enterprise Award for entrepreneurship. He is the only two-time $(2000,2004)$ Developer of the Year Award winner from the Software Council of Southern California, and Computerworlds selection to the elite 100 Emerging Companies to Watch in 2000. He is the recipient of the National Leadership Award and 2003 Businessman of the Year Award for California from the Business Advisory Council of the National Congressional Committee, and serves as its Honorary Co-Chairman for California. His research has been sponsored by numerous prestigious organizations including DARPA, OSD, ONR, NAVSEA, NAVAIR, NRL, AFOSR, AFRL, Tinker AFB, CECOM, AMCOM, ARI, HEL, NIST, DOC, DOE, and NASA. He has received special commendations from DARPA, the Office of the Secretary of Defense, the U.S. Navy, and the U.S. SBA for his outstanding contributions to national agility and concurrent engineering initiatives. From 1977-1994, he was the Executive Vice-President and Chief Technology Officer of Perceptronics, Inc., a public company where he pioneered groundbreaking advances in process modeling and distributed simulation technology for human-system integration and distributed training.

Copyright (C) 2010 by the author. This is an open access article distributed under the Creative Commons Attribution License (https://creativecommons.org/licenses/ by/4.0/), which permits unrestricted use, distribution, and reproduction in any medium, provided the original work is properly cited. 